\title{
Accelerating de novo drug design against novel proteins using deep
}

\section{learning}

Sowmya Ramaswamy Krishnan*, Navneet Bung", Gopalakrishnan Bulusu* and Arijit Roy*

TCS Innovation Labs-Hyderabad (Life Sciences Division), Tata Consultancy Services

Limited, Hyderabad 500081, India.

${ }^{\#}$ Authors with equal contribution

*Authors for Correspondence: g.bulusu@tcs.com and roy.arijit3@tcs.com

\section{Supporting information 1}

\section{Pre-trained generative model learned the SMILES grammar}

The pre-trained generative model was trained with the SMILES strings of $\sim 1.6$ million drug-like small molecules from the ChEMBL database ${ }^{1}$. The model was found to have a high accuracy of $96.6 \%$ defined as the mean percentage of chemically valid molecules present in all the sampling batches. About $1.2 \%$ of the generated valid small molecules were identical to the training dataset and $2.2 \%$ of the molecules were redundant. The internal diversity of the sampled molecules measured in units of Tanimoto distance ${ }^{2}$ was found to be 0.70 , indicating high internal diversity. The baseline metrics of the pre-trained generative model including validity, novelty, Fréchet ChemNet Distance (FCD) score, and Kullback-Leibler (KL) divergence score of various physico-chemical property distributions in comparison with the training dataset were calculated using the GuacaMol benchmark guidelines ${ }^{3}$. The lower synthetic accessibility scores ${ }^{4}$ for the generated small molecules indicate the ease of synthesis. The baseline evaluation metrics (discussed below) and the desirable physico-chemical properties of the generated small molecules shows similar distribution to the drug-like molecules obtained from the ChEMBL database (Figure S1).

\section{Metrics of the pre-trained generative model}

The pre-trained generative model was evaluated using the metrics specified in the earlier studies ${ }^{3}$.

The generative model was found to have a high accuracy of $96.6 \%$ defined as the validity of the generated small molecules. The validity was verified using the RDKit cheminformatics library ${ }^{4}$ in Python. The uniqueness of the model was 0.9987 , indicating that $99 \%$ of the 10,000 molecules sampled from the pre-trained model were unique. The novelty score of the model is 0.9815 , where only $2 \%$ of the 10,000 molecules sampled were found to be present in the training dataset from the ChEMBL database ${ }^{1}$. The $\mathrm{KL}$ divergence metric for 9 physicochemical descriptors compared with the training dataset was found to be 0.991 . This shows that the model has accurately captured the physicochemical property distributions of drug-like small molecules during pre-training. The Fréchet ChemNet Distance (FCD) of the sampled set and a random subset of 10,000 molecules from the training dataset was 0.728. Lower FCD scores indicate a lower similarity between the training set and the molecules from the generative model thereby, suggesting that the model has not memorized the small molecules from the training dataset. Thus, all the metrics of our pre-trained generative model are in accordance with the desirable thresholds for the benchmark dataset (from the ChEMBL database).

\section{Overview of the Janus kinase family of proteins}


The human Janus kinase (JAK) family of proteins play a very essential role in the innate and adaptive immune systems, by interacting with cytokines through the JAK/STAT signaling pathway ${ }^{6}$. They are non-receptor tyrosine kinase proteins involved in cell growth, survival, development and differentiation of a variety of cells, apart from immune cells and hematopoietic cells. Activation of JAK proteins through interaction with cytokines leads to several downstream signaling events mediated by the activation of STAT proteins (signal transducers and activators of transcription). STAT proteins are cytosolic DNA-binding proteins which can regulate the transcription of several downstream genes constituting the JAK/STAT signaling pathway. Multiple mutations in the four proteins of the JAK family namely, JAK1, JAK2, JAK3 and TYK2, have been implicated in various immune disorders and cancers ${ }^{7}$.

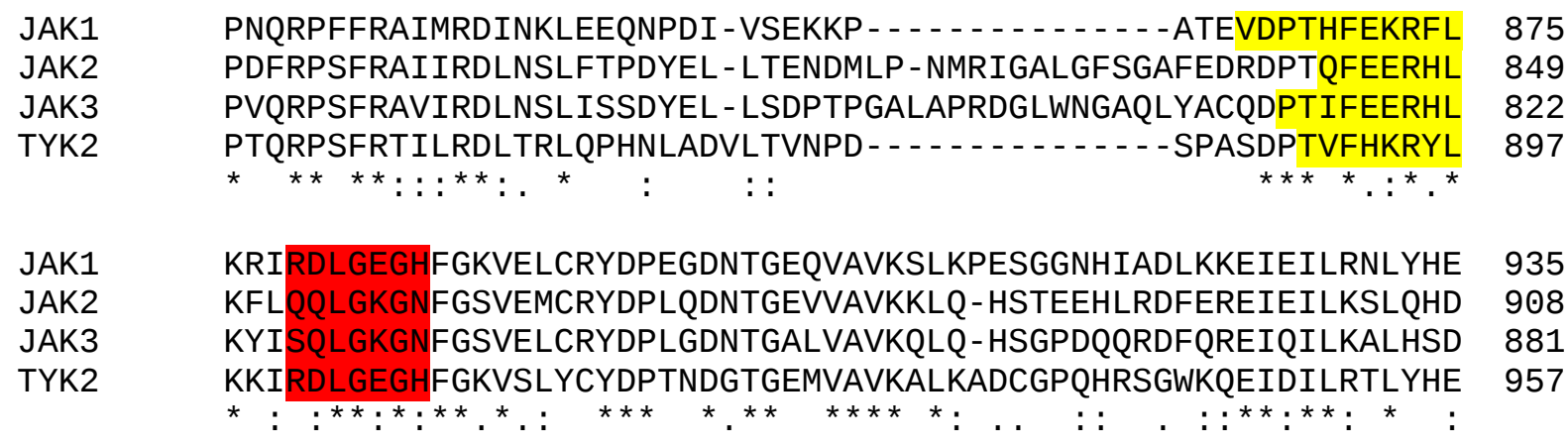

JAK1 NIVKYKGICTEDGGNGIKLIMEFLPSGSLKEYLPKNKNKINLKQQLKYAVQICKGMDYLG

JAK2

JAK3

TYK2 NIVKYKGVCYSAGRRNLKLIMEYLPYGSLRDYLQKHKERIDHIKLLQYTSQICKGMEYLG FIVKYRGVSYGPGRQSLRLVMEYLPSGCLRDFLQRHRARLDASRLLLYSSQICKGMEYLG HIIKYKGCCEDQGEKSLQLVMEYVPLGSLRDYLPRH - SIGLAQLLLFAQQICEGMAYLH ${ }^{*}{ }^{*}:{ }^{*} .::^{*}:{ }^{*}::^{*}{ }^{*}{ }^{*}::^{*}:: \quad::^{*}::^{* * *}:{ }^{*}{ }^{* *}$

SRQYVHRDLAARNVLVESEHQVKIGDFGLTKAIETDKEYYTVKDDRDSPVFWYAPECLMQ TKRYIHRDLATRNILVENENRVKIGDFGLTKVLPQDKEYYKVKEPGESPIFWYAPESLTE SRRCVHRDLAARNILVESEAHVKIADFGLAKLLPLDKDYYVVREPGQSPIFWYAPESLSD AQHYIHRDLAARNVLLDNDRLVKIGDFGLAKAVPEGHEYYRVREDGDSPVFWYAPECLKE

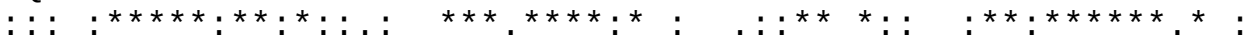

SKFYIASDVWSFGVTLHELLTYCDSDSSPMALFLKMIGPT - HGQMTVTRLVNTLKEGKRL SKFSVASDVWSFGVVLYELFTYIEKSKSPPAEFMRMIGNDKQGQMIVFHLIELLKNNGRL NIFSRQSDVWSFGVVLYELFTYCDKSCSPSAEFLRMMGCERDVP - ALCRLLELLEEGQRL YKFYYASDVWSFGVTLYELLTHCDSSQSPPTKFLELIGIA-QGQMTVLRLTELLERGERL

$\begin{array}{lll}\text { JAK1 } & --- & 1154 \\ \text { JAK2 } & --- & 1132 \\ \text { JAK3 } & \text { LSFS } & 1124 \\ \text { TYK2 } & ---- & 1187\end{array}$

The multiple sequence alignment (MSA) of the sequence of the kinase domains of the four JAK family proteins is shown above. The MSA was generated using the Clustal Omega (v1.2.4) program $^{8}$. The protein sequences were collected from UniProt ${ }^{9}$ (JAK1-P23458, JAK2-O60674, JAK3-P52333, TYK2-P29597). The sequence highlighted in yellow indicates the start of the kinase domain of the three proteins based on the crystal 
structures from Protein Data Bank (JAK1 - PDB ID: 6N7A, JAK2 - PDB ID: 4IVA, JAK3 PDB ID: 5LWM, TYK2 - PDB ID: 4GFO). The region of the sequence highlighted in red indicates the sequentially discontinous active site of the protein. Although the structures of the four kinase domains could be aligned with < $1.1 \AA$ RMSD (see Fig. S2 below), the sequence of the active site indicates the residue-level variations between the three proteins.

Although the overall fold of all the four proteins is highly conserved, there are minor changes in the active site. Crystal structures of all the JAK family kinase domains have been determined. These structures contain a $\beta$-sheet $\mathrm{N}$-lobe that is conformationally flexible (facilitating activation regulation and ATP/ADP binding and release), a nucleotidebinding glycine-rich loop (P-loop), an extended activation loop which is phosphorylated in almost all kinases, and a DFG motif. All small molecule inhibitors bind in the ATP-cleft juxtaposed between a $\beta$-sheet-rich $\mathrm{N}$-lobe and the $\alpha$-helix-rich $\mathrm{C}$-lobe. The major conformational differences are centered on the glycine rich loop ( $\mathrm{P}$-loop) and the activation loop. In the region of the ATP-binding site, only two residues are divergent within $6 \AA$ between the JAK family members. In JAK1, JAK2, and TYK2 these residues are serine and glycine, while in JAK3 they are cysteine and alanine, respectively ${ }^{10}$.

It has been identified through experimental studies that the presence of a cysteine instead of a serine in the active site (residue 909) of JAK3, enables selectivity of the ligands through covalent interactions ${ }^{12-14}$. Several inhibitors have been designed and verified through experiments for selective binding to the active site of any one of the JAK family proteins $^{10-14}$. Studies have established the role of Cys909 in the selectivity of covalent ligands towards JAK3. Similarly, the residues and molecular subgroups providing selectivity towards each JAK family protein have been identified to some extent ${ }^{6,7}$. The non-redundant functionality of the proteins of this family in activation of the JAK/STAT pathway and several other downstream pathways, indicates their essential role in immune response ${ }^{6}$. Hence, it is essential that we design inhibitors specific to each protein to better understand their selective mechanism of activation and other downstream signaling events.

\section{Comparison of features of the JAK2-selective molecules and the generated molecules}

Based on the literature and the assay data for the patented molecules, the structural features necessary for JAK2 selectivity over JAK1, JAK3 and TYK2 proteins were enumerated $^{15-21}$. Some of these features include - presence of aminopyrazole ${ }^{22}$ (Fig. S4b), aminothiazole ${ }^{23}$ (Fig. S4-C), triazolo pyridine ${ }^{24}$ (Fig. S4-e), or diaminopyrimidine ${ }^{25}$ (Fig. S4-j) in the head group. The presence of cyclohexanol group ${ }^{26}$ (Fig. S4-a), morpholine group $^{27}$ (Fig. S4-d), 4-fluorobenzyl group ${ }^{28}$ (Fig. S4-f), dicyclopropyl group ${ }^{29}$ (Fig. S4-g), cyano/nitrile ${ }^{16}$ (Fig. S4-h), carboxamide group ${ }^{29}$ (Fig. S4-i), and trifluoromethyl group ${ }^{30}$ (Fig. S4-k) have also been shown to provide higher JAK2 selectivity. Based on these features guiding JAK2 selectivity identified from literature, we were able to identify several generated small molecules which have the above mentioned functional groups. Some of the identified small molecules with the selectivity feature are shown in Fig. S4.

\section{Generative model can generate novel scaffolds by exploring the chemical space}

The unique Murcko scaffolds ${ }^{31}$ present in the training set, validation set, and the filtered set of molecules from the generative model were extracted using the built-in function in the RDKit library ${ }^{2}$. The total number of unique Murcko scaffolds were found to be 1,406 in 
training set, 537 in validation set and 6,295 among the molecules generated by the model. In order to extract the novel JAK2 scaffolds generated by the model, the 6,295 scaffolds were compared with the scaffolds from the validation set. A Tanimoto coefficient cut-off of 0.5 was used to define dissimilarity between a pair of scaffolds ${ }^{32}$. After all pair-wise scaffold similarities were computed, 2,461 scaffolds (39.09\%) from the generated molecules were found to be dissimilar to the scaffolds in the validation set.

However, it is also possible that these 2,461 scaffolds might be present in the training set molecules. Based on comparison with the scaffolds from the training dataset it was identified that, 1,587 scaffolds $(25.21 \%$ of total 6,295$)$ from the generated small molecules were dissimilar to the training set scaffolds.

In order to group the similar scaffolds among the 1,587 novel scaffolds, butina clustering ${ }^{33}$ was performed with Tanimoto coefficient as the distance metric using RDKit. Based on the clustering results, scaffold clusters with minimum cluster size of 5 were chosen and the representative scaffold of the cluster was extracted. Some of these novel JAK2 scaffolds extracted from the filtered set of generated molecules are shown in Fig. S5.

\section{Effectiveness of using both transfer and reinforcement learning to train the generative model}

In order to understand the effectiveness of using both transfer and reinforcement learning to train the generative model, we further performed additional tests using only transfer learning and only reinforcement learning, respectively and compared them with the results from the Fig. 2(a) and 2(b) of the manuscript.

\section{Only transfer learning}

The training dataset obtained after docking the JAK1, JAK3 and TYK2 inhibitors was used for transfer learning. After 100 epochs of transfer learning, the distribution of the maximum Tanimoto coefficient between the molecules from the generative model was found to have a good shift compared to the distribution before transfer learning (Fig. S6a). Post-transfer learning, 10,000 molecules were sampled from the generative model. Redundant and ChEMBL-identical molecules were removed, and the same set of filters (physico-chemical property filters and rule-based filters) were applied on the sampled set of molecules. Further, only molecules with $<=-7.0$ docking score were considered for further analysis.

While comparing the molecules with the validation dataset (JAK2 inhibitors) it was found that, 339 molecules had >= 0.75 maximum Tanimoto similarity to the molecules from the validation dataset. However, the predicted docking score distribution was close to the model before transfer learning (Fig. S6b). Therefore, the generative model with only transfer learning could not generate molecules with high binding affinity to the JAK2 active site, although it could generate molecules with scaffold similarity to the existing JAK2 inhibitors.

\section{Only reinforcement learning}

The pre-trained generative model was directly subjected to docking score optimization with the predictive model without the intermediate transfer learning step. With the objective of optimizing the docking score distribution, the model was trained with reinforcement learning for 80 epochs, based on the shift in the docking score distribution. The resultant generative model was used to sample 10,000 small molecules and these molecules were subject to the same set of filters as mentioned above.

On comparing the filtered set of molecules with the existing JAK2 inhibitors, it was found that only 13 molecules had $>=0.75$ maximum Tanimoto similarity with the validation dataset. From this observation it can be inferred that, while the docking score distribution 
is being optimized (Fig. S6d), the similarity of the generated molecules to the validation dataset is very poor (Fig. S6c). Even with a training of up to 500 epochs of reinforcement learning, the similarity distribution could not be improved. This emphasizes the need for a combined training technique with both transfer learning and reinforcement learning.

\section{Unique generated scaffolds present in the validation dataset but absent in the training dataset}

In order to understand if there is any unique scaffold generated by the algorithm that is common with the validation dataset but absent in the training dataset, Tversky similarity was used. A previous study ${ }^{34}$ has extensively reviewed the importance of choosing the appropriate $\alpha$ and $\beta$ values for sub-structure mapping with Tversky similarity. Based on that study ${ }^{34}$ and by testing multiple values, the final values of $\alpha$ and $\beta$ were fixed at 0.9 and 0.1 , respectively. In order to identify the scaffolds unique to the validation dataset, the 537 Murcko scaffolds from the validation dataset were compared with the 1,406 Murcko scaffolds from training dataset using RDKit. With a Tversky similarity cut-off of $<0.75,146$ scaffolds from the validation dataset were found to be unique compared to the training set scaffolds.

The 6,295 unique Murcko scaffolds from the final set of generated small molecules were further compared with these 146 scaffolds unique to the validation dataset. 131 scaffolds from the generated molecules were found to have $>=0.90$ Tversky similarity indicating high similarity to the unique JAK2 scaffolds. A representative set of the generated scaffolds with high similarity to the unique validation dataset scaffolds is shown in the Table S3 below. 
Table S1: Details of the architecture and hyperparameters used for training the generative and predictive models.

\begin{tabular}{|c|c|c|c|c|}
\hline Hyperparameter & $\begin{array}{c}\text { Generative } \\
\text { model (Prior) }\end{array}$ & $\begin{array}{c}\text { Predictive } \\
\text { model (Critic) }\end{array}$ & $\begin{array}{l}\text { Transfer } \\
\text { learning }\end{array}$ & $\begin{array}{c}\text { Reinforcement } \\
\text { learning } \\
\text { (Agent) }\end{array}$ \\
\hline $\begin{array}{c}\text { Type of neural } \\
\text { network }\end{array}$ & $\begin{array}{c}\text { Recurrent } \\
\text { neural network } \\
\text { (RNN) }\end{array}$ & $\begin{array}{c}\text { Recurrent } \\
\text { neural network } \\
\text { (RNN) }\end{array}$ & $\begin{array}{c}\text { Recurrent } \\
\text { neural network } \\
\text { (RNN) }\end{array}$ & $\begin{array}{c}\text { Recurrent neural } \\
\text { network (RNN) }\end{array}$ \\
\hline No. of layers & 1 & 3 & 1 & 1 \\
\hline Bidirectional layer & Yes & Yes & Yes & Yes \\
\hline $\begin{array}{l}\text { Type of internal } \\
\text { memory cell }\end{array}$ & $\begin{array}{c}\text { Gated } \\
\text { Recurrent Unit } \\
(\text { GRU })\end{array}$ & $\begin{array}{c}\text { Gated } \\
\text { Recurrent Unit } \\
(\text { GRU })\end{array}$ & $\begin{array}{c}\text { Gated } \\
\text { Recurrent Unit } \\
(\text { GRU })\end{array}$ & $\begin{array}{l}\text { Gated Recurrent } \\
\text { Unit (GRU) }\end{array}$ \\
\hline Hidden layer size & 1024 & 256 or 128 & 1024 & 1024 \\
\hline $\begin{array}{l}\text { Type of external } \\
\text { memory cell }\end{array}$ & Stack & NA & Stack & Stack \\
\hline $\begin{array}{l}\text { External memory } \\
\text { cell dimensions }\end{array}$ & $512 \times 100$ & NA & $512 \times 100$ & $512 \times 100$ \\
\hline $\begin{array}{l}\text { Activation between } \\
\text { hidden layers }\end{array}$ & ReLU & ReLU & ReLU & ReLU \\
\hline $\begin{array}{l}\text { Activation at final } \\
\text { dense layer }\end{array}$ & Softmax & ReLU & Softmax & Softmax \\
\hline Initial learning rate & 0.0005 & 0.001 & 0.0005 & 0.00005 \\
\hline Batch size & 256 & 64 & 256 & 256 \\
\hline Dropout rate & 0.2 & 0.3 & 0.2 & 0.2 \\
\hline Optimizer & AMSGrad & Adam & AMSGrad & AMSGrad \\
\hline $\begin{array}{l}\text { Learning rate } \\
\text { decay }\end{array}$ & Yes & Yes & Yes & Yes \\
\hline Gradient clipping & Yes & No & Yes & Yes \\
\hline No. of epochs & $300-400$ & $300-500$ & $50-100$ & $30-100$ \\
\hline GPU details & $\begin{array}{c}1 \text { Tesla V100 } \\
\text { GPU }\end{array}$ & $\begin{array}{c}1 \text { Tesla V100 } \\
\text { GPU }\end{array}$ & $\begin{array}{c}1 \text { Tesla V100 } \\
\text { GPU }\end{array}$ & $\begin{array}{c}1 \text { Tesla V100 } \\
\text { GPU }\end{array}$ \\
\hline $\begin{array}{l}\text { Implementation } \\
\text { framework }\end{array}$ & PyTorch & PyTorch & PyTorch & PyTorch \\
\hline
\end{tabular}

Table S2: Percentage of molecules which passed each of the rule-based filters and the common errors flagged by each filter for the input set of molecules from the generative model.

\begin{tabular}{|c|c|c|}
\hline $\begin{array}{c}\text { Rule-based } \\
\text { filter }\end{array}$ & $\begin{array}{c}\text { Qualified } \\
\text { molecules } \\
\mathbf{( \% )}\end{array}$ & $\begin{array}{c}\text { Common sub-structural flags (Rule notations as } \\
\text { defined in RDKit) }\end{array}$ \\
\hline PAINS & 96.51 & $\begin{array}{c}\text { anil_di_alk, anil_NH_alk, azo, catechol, ene_cyano, } \\
\text { ene_five_het, imine_one, mannich }\end{array}$ \\
\hline
\end{tabular}




\begin{tabular}{|c|c|c|}
\hline BRENK & 78.06 & $\begin{array}{l}\text { 2-halo_pyridine, aldehyde, Aliphatic_long_chain, } \\
\text { alkyl_halide, aniline, beta-keto/anhydride, } \\
\text { Carbo_cation/anion, catechol, } \\
\text { charged_oxygen_or_sulfur_atoms, } \\
\text { conjugated_nitrile_group, cumarine, cyanamide, } \\
\text { cyanate_/aminonitrile_/thiocyanate, diazo_group, } \\
\text { diketo_group, halogenated_ring_1, het-C-het_not_in_ring, } \\
\text { hydantoin, hydroquinone, hydroxamic_acid, Oxygen- } \\
\text { nitrogen_single_bond, imine_1, isolated_alkene, } \\
\text { Michael_acceptor_1, N-C-halo, nitro_group, Perfluorinated } \\
\text { chain,phosphor, pthalimide, } \\
\text { Polycyclic_aromatic_hydrocarbon_3, stilbene, } \\
\text { quarternary_nitrogen_1, Sulfonic_acid_1, } \\
\text { Thiocarbonyl_group, thioester, Three-- } \\
\text { membered_heterocycle, triple_bond, }\end{array}$ \\
\hline $\mathrm{NIH}$ & 94.32 & $\begin{array}{l}\text { 4halo_pyridine_3EWG, acrylate, halo_acrylate, aldehyde, } \\
\text { alpha_dicarbonyl, alpha_halo_carbonyl, } \\
\text { primary_halide_sulfate, aziridine_diazirine, betalactam, } \\
\text { cyanohydrin, halogen_heteroatom, sulfonyl_halide, } \\
\text { hydrazine, hydroxamate_warhead, isonitrile, } \\
\text { non_ring_CH2O_acetal, primary_halide_sulfate, } \\
\text { trisub_bis_act_olefin }\end{array}$ \\
\hline ZINC & 96.68 & $\begin{array}{l}\text { Acid_halides, P/S_halides, Fluorines, Isonitriles, Non- } \\
\text { Hydrogen_atoms }\end{array}$ \\
\hline
\end{tabular}

Table S3: A representative set of the generated scaffolds with high similarity to the unique validation set scaffolds. The similarity between scaffolds has been quantified using Tversky similarity (TS). The first column shows unique scaffolds that are common between the generated and validation datasets but absent in the training dataset. The second column shows the closest matching scaffold in the training dataset with respect to the same validation set scaffold.

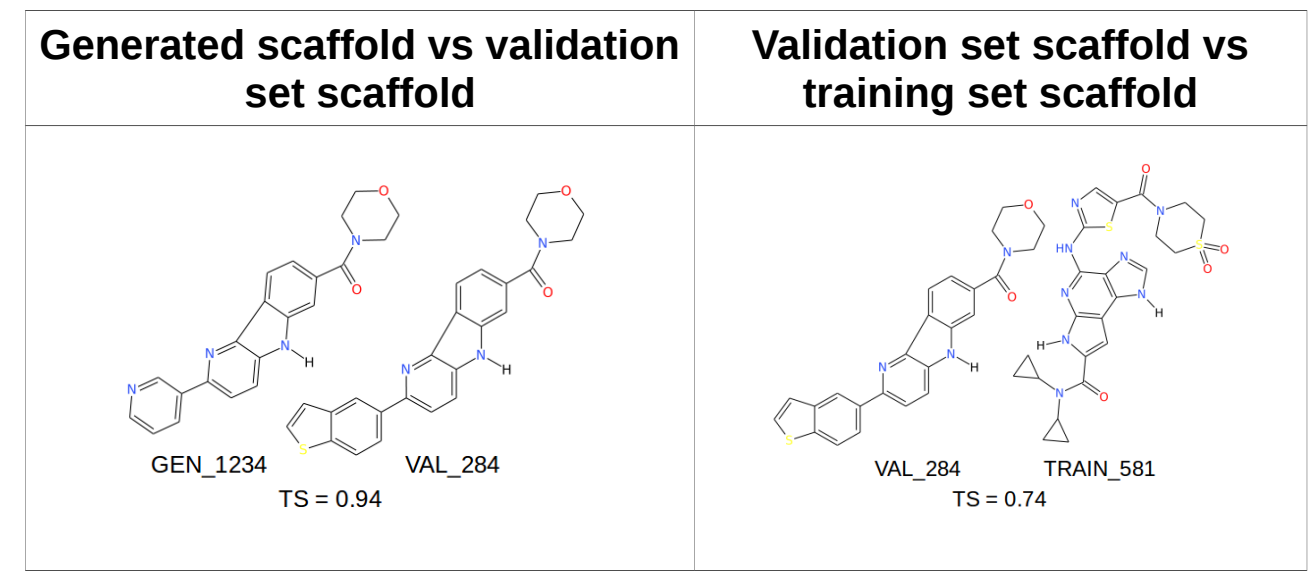




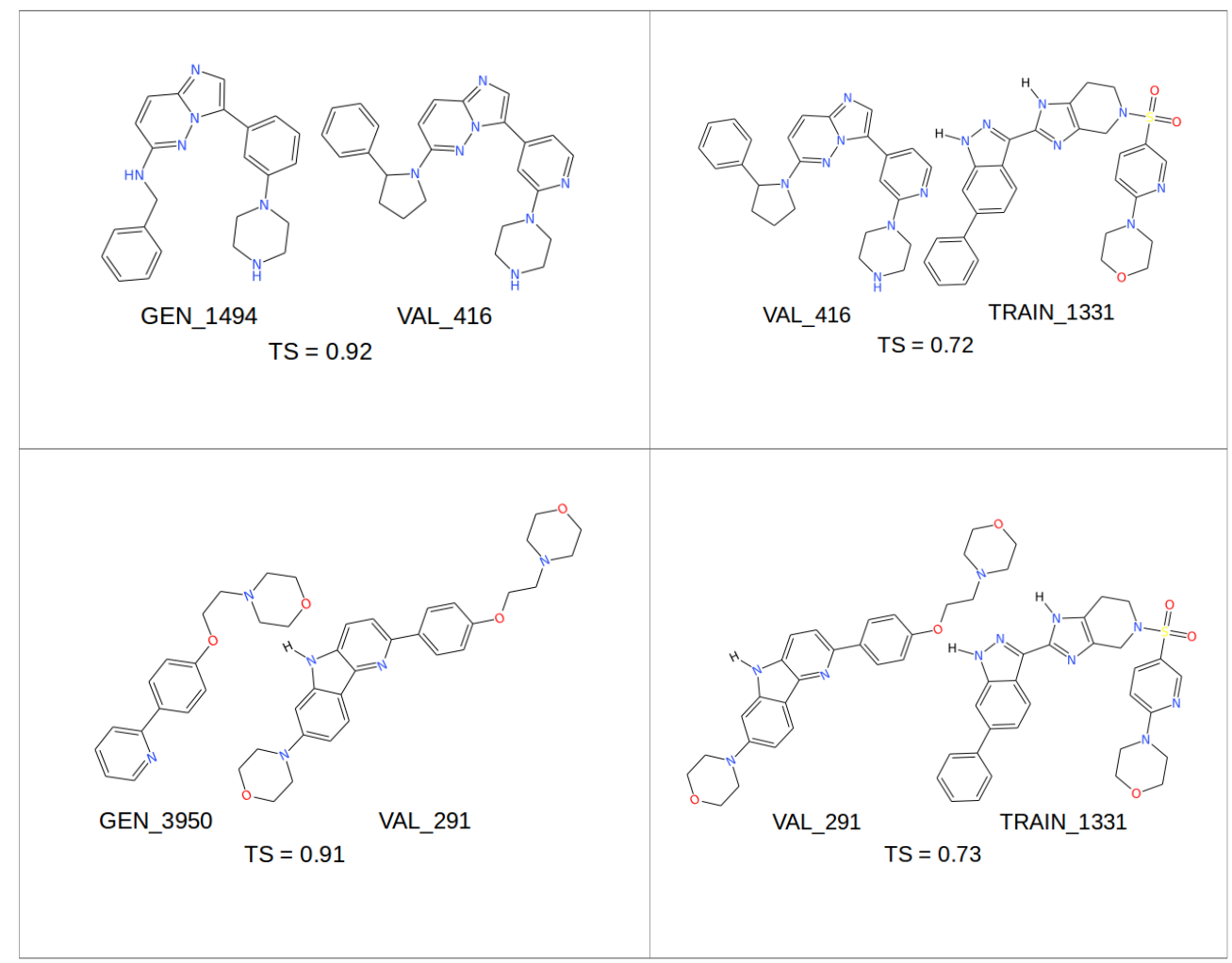




\section{Supplementary figures}

a
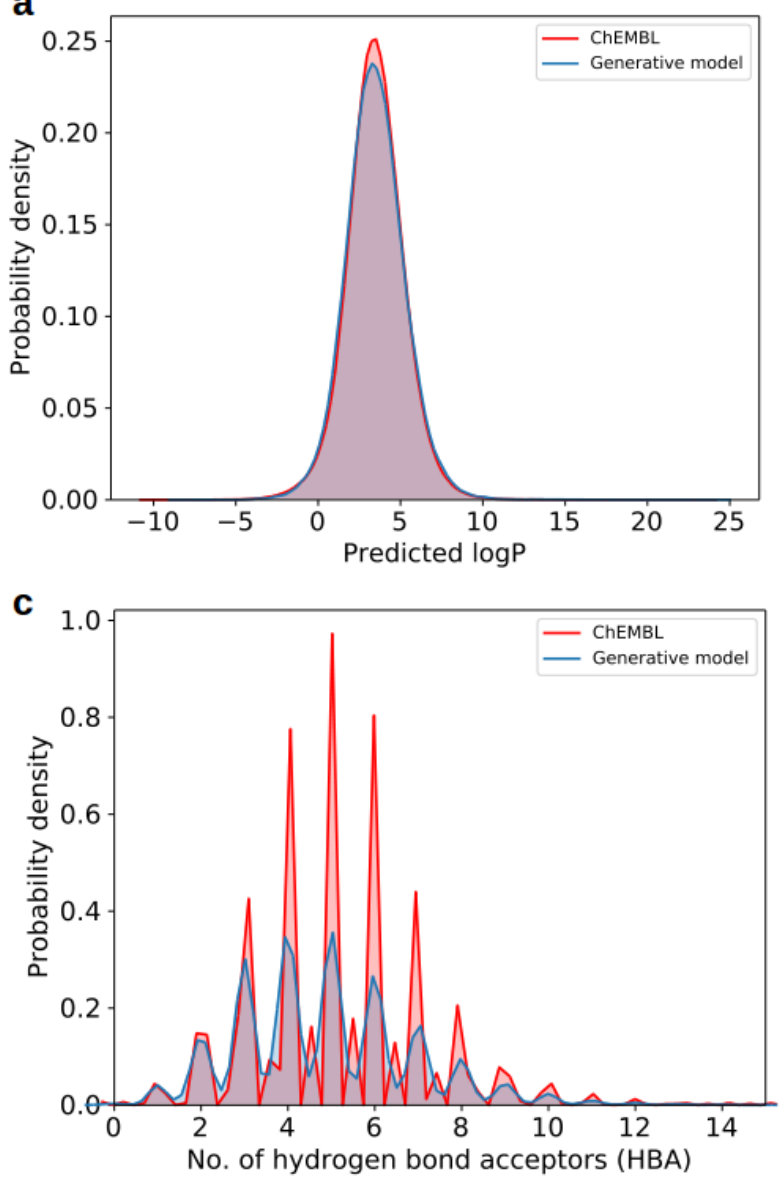

e

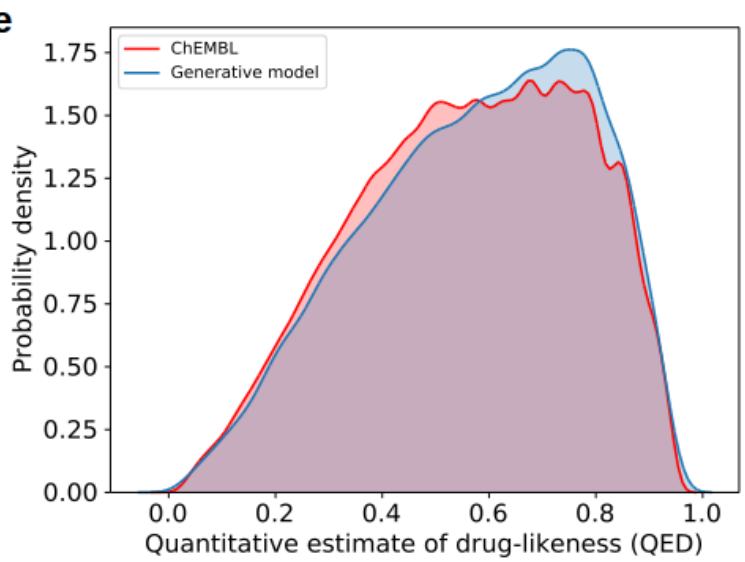

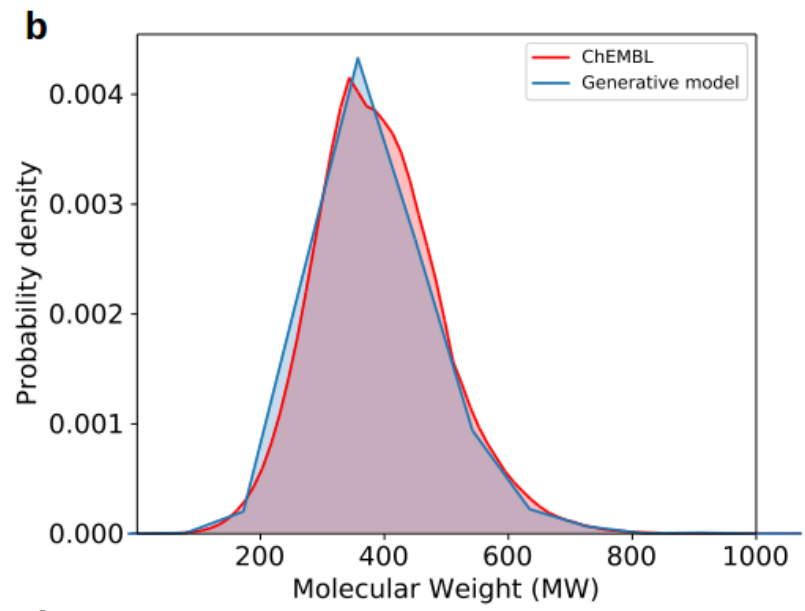

d

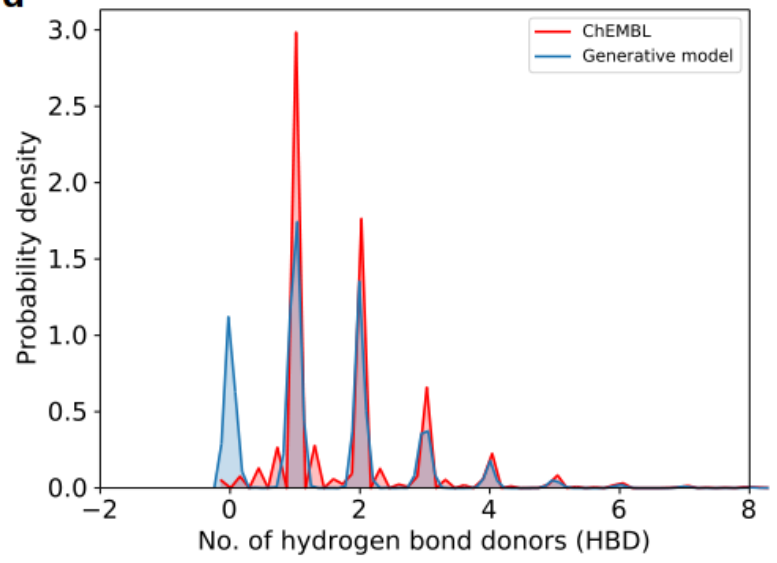

f

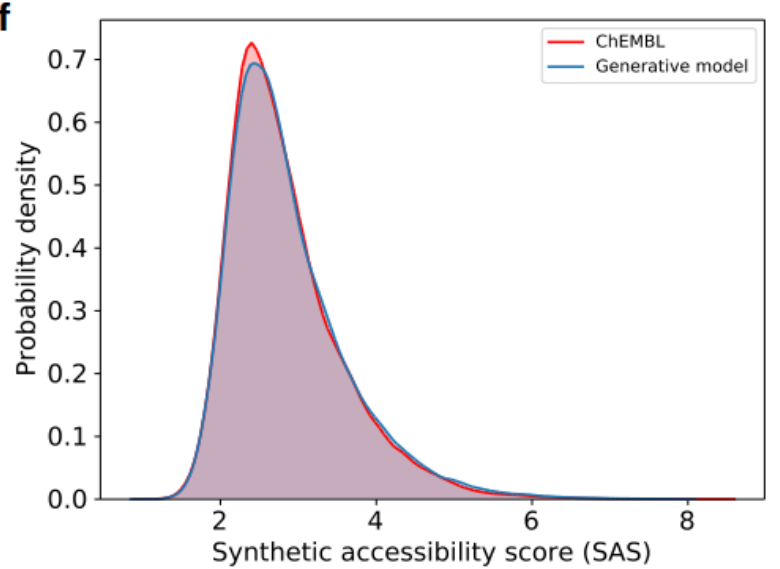

Figure S1: The physico-chemical property distributions of the pre-trained generative model compared with the distributions for the training dataset from the ChEMBL database. a Octanol-water partition coefficient $(\log P)$, b Molecular weight $(\mathrm{MW})$, c Number of hydrogen bond acceptors (HBA), d Number of hydrogen bond donors (HBD), e Quantitative estimate of drug-likeness (QED) ${ }^{35}$ and $\mathbf{f}$ Synthetic accessibility score (SAS) ${ }^{4}$. 


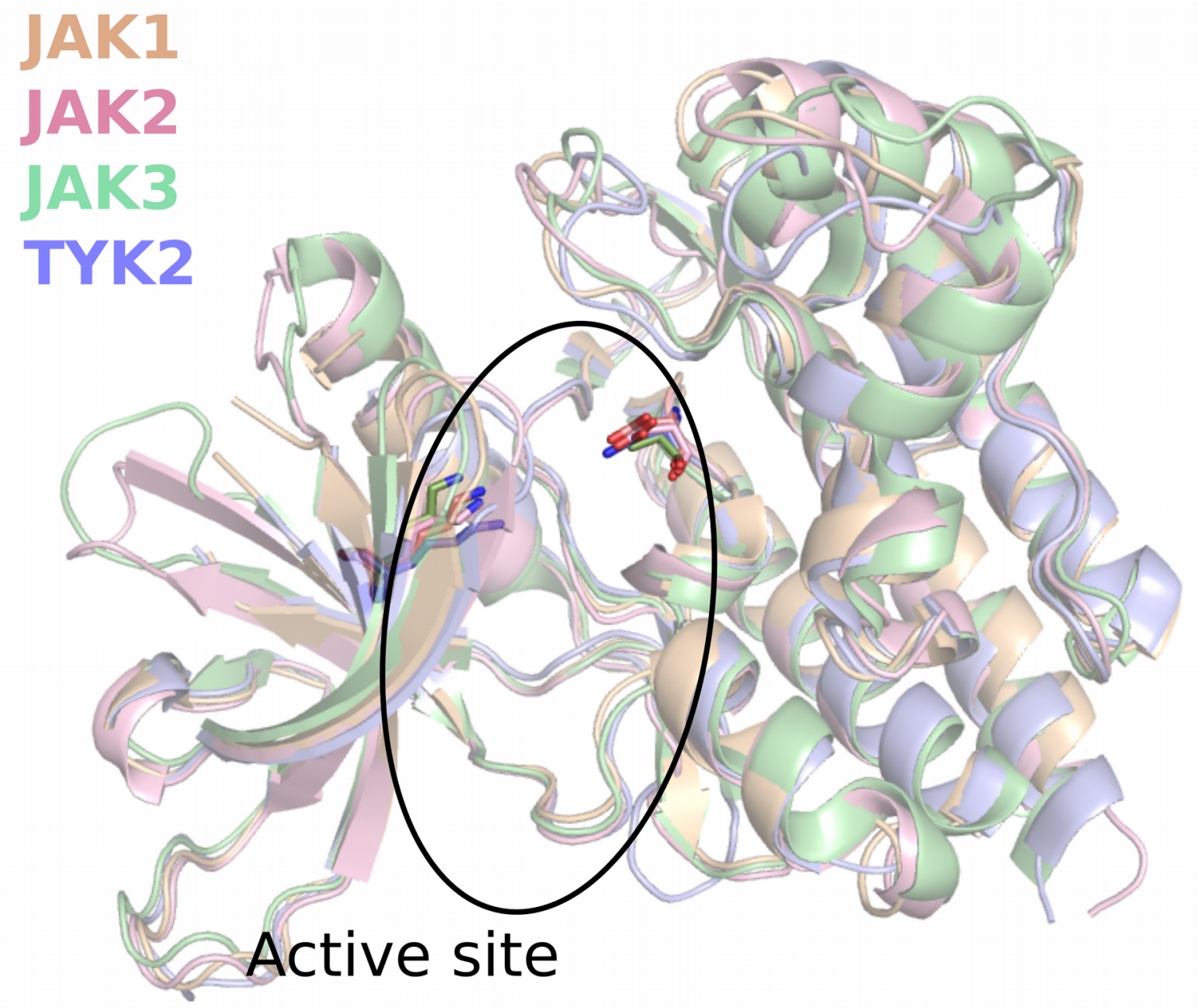

Figure S2: Alignment of the crystallographic structures of the kinase domains of the proteins from the Janus kinase family - JAK1 (PDB ID: 6N7A), JAK2 (PDB ID: 4IVA), JAK3 (PDB ID: 5LWM) and TYK2 (PDB ID: 4GFO). Two conserved residues Lys and Asp are shown in sticks. 

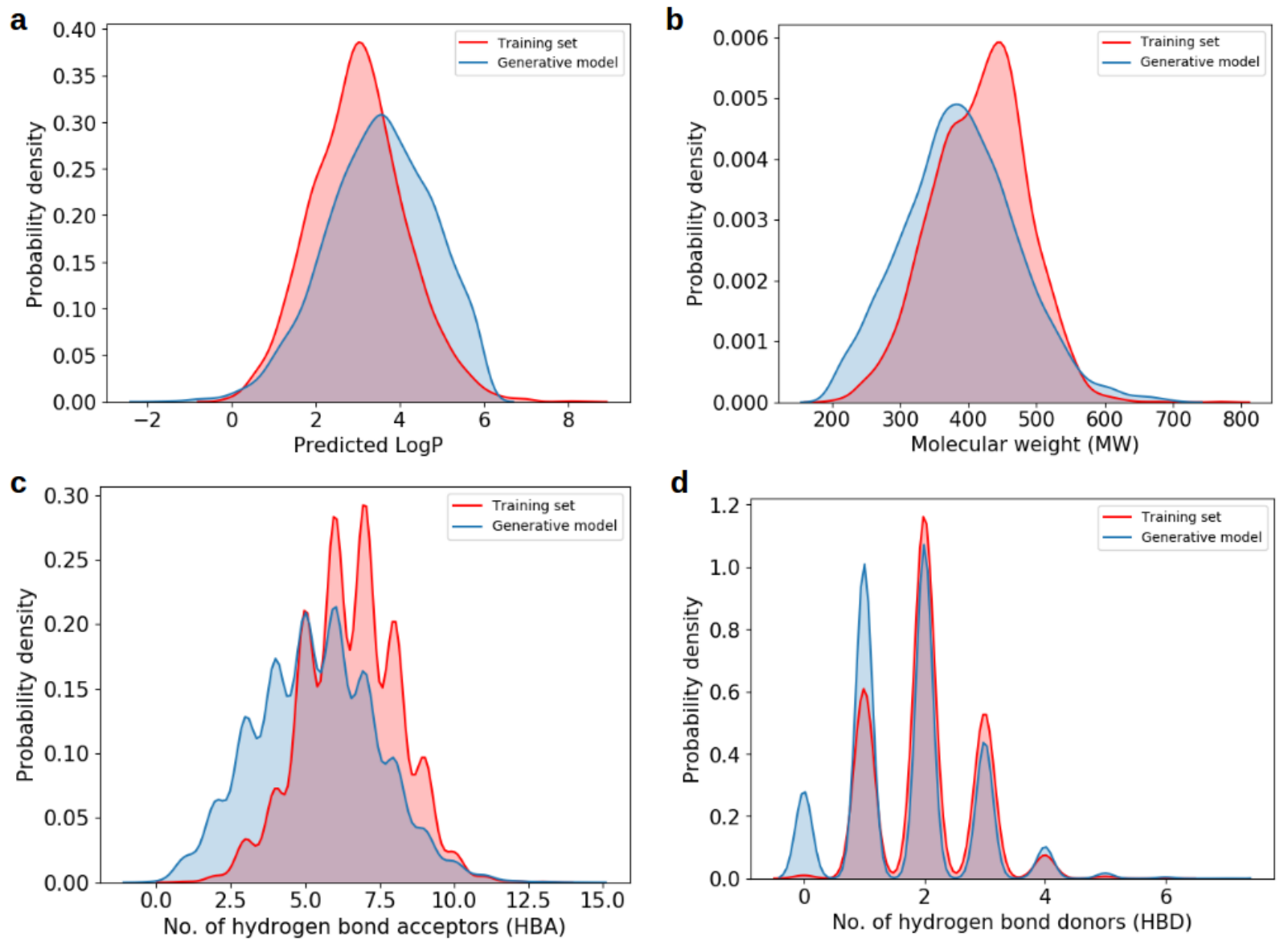

Figure S3: The physico-chemical property distributions of the generative model obtained after TL and RL, compared with the distributions for the TL training dataset. a Octanolwater partition coefficient $(\log P)$, b Molecular weight $(\mathrm{MW})$, c Number of hydrogen bond acceptors (HBA), and $\mathbf{d}$ Number of hydrogen bond donors (HBD). 


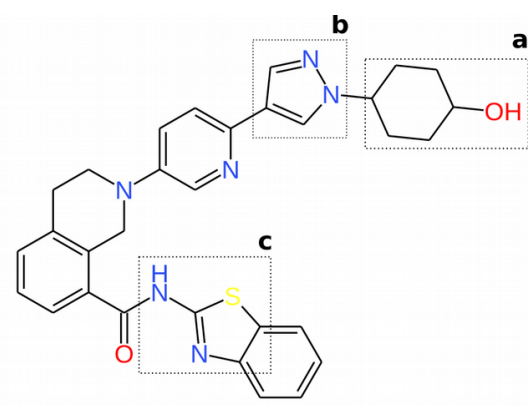

Mol_1285 (-11.1)

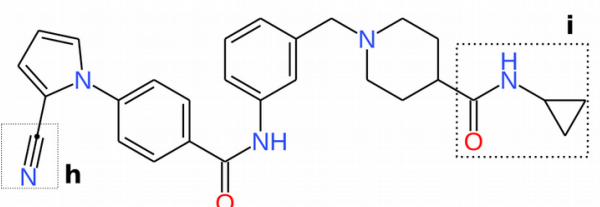

Mol_3745 (-9.9)

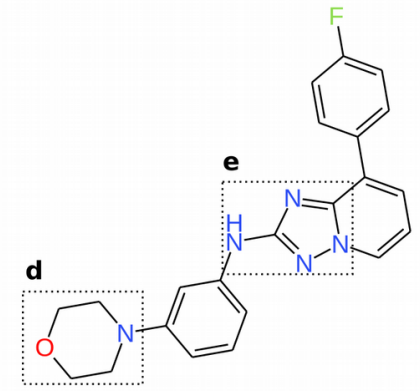

Mol_4171 (-10.2)

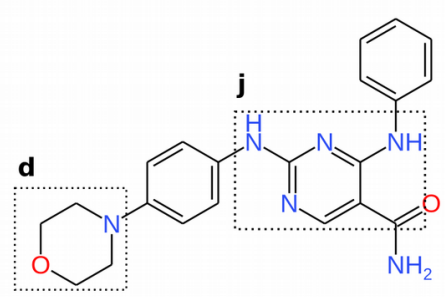

Mol_4408 (-9.5)

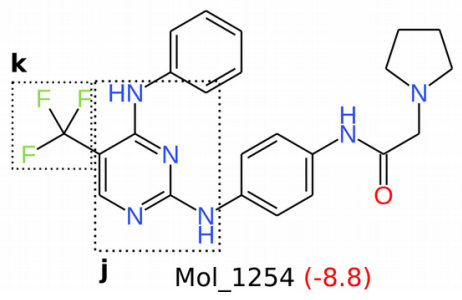

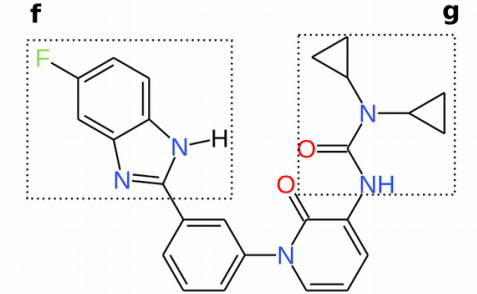

Mol_5364 (-9.9)

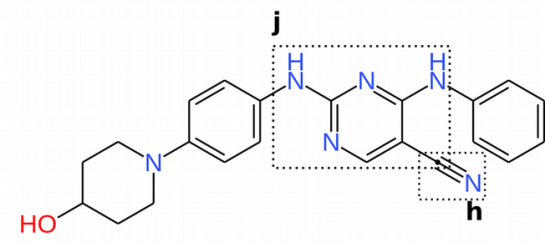

Mol_5982 (-9.4)

Figure S4: A representative set of generated small molecules after reinforcement learning. The actual docking score of the small molecule is shown in red. The sub-groups and head groups that are known to increase selectivity towards JAK2 active site are highlighted ( $\mathbf{a}-$ Cyclohexanol, b - Pyrazole, c - Aminothiazole, $\mathbf{d}$ - Morpholine, e - Triazolo pyridine, $\mathbf{f}$ 4-fluorobenzyl group, $\mathbf{g}$ - Dicyclopropyl carboxamide, $\mathbf{h}$ - Nitrile/Cyano group, i Cyclopropyl carboxamide, $\mathbf{j}$ - Diaminopyrimidine, $\mathbf{k}$ - Trifluoromethyl group). 
a<smiles>O=C(Nc1ccccc1)N1CCC(c2nccn2CC2CC2)CC1</smiles>

d<smiles>O=C(Nc1ccccc1)Nc1cccc(-c2cccc(Nc3ccccc3)c2)c1</smiles>

b<smiles>c1ccc2sc(Nc3nc4ccccc4s3)nc2c1</smiles>

C<smiles>O=C(Nc1ccccc1)Nc1ccc(-c2nc3ccccc3o2)cc1</smiles>

e<smiles>c1cnc(Nc2ccc(Nc3nc4ccccc4[nH]3)cc2)nc1</smiles>

Figure S5: A subset of the novel representative JAK2 scaffolds generated by the generative model, identified based on butina clustering. 
a

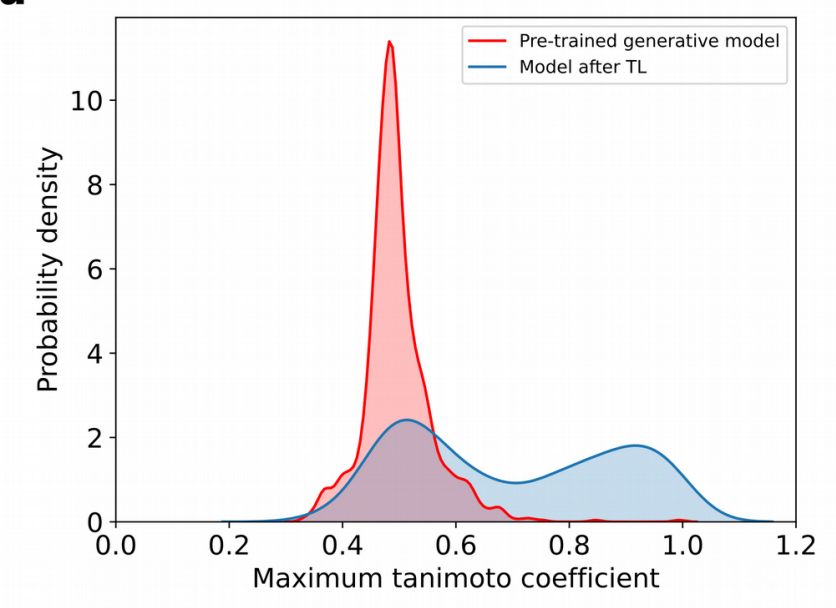

C

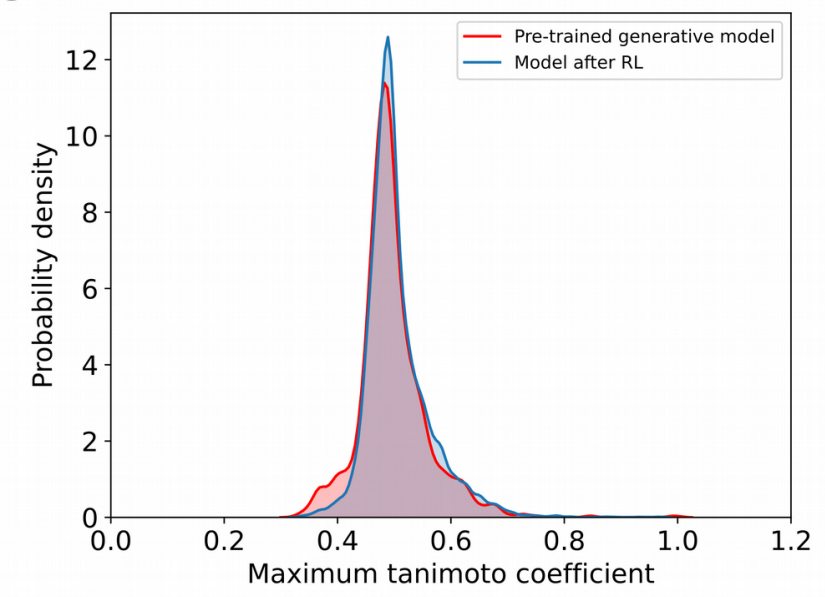

b

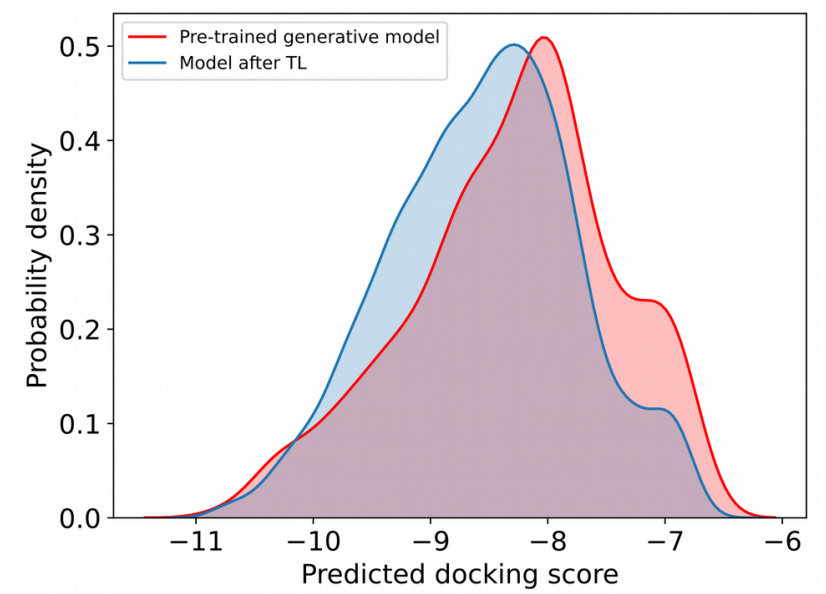

d

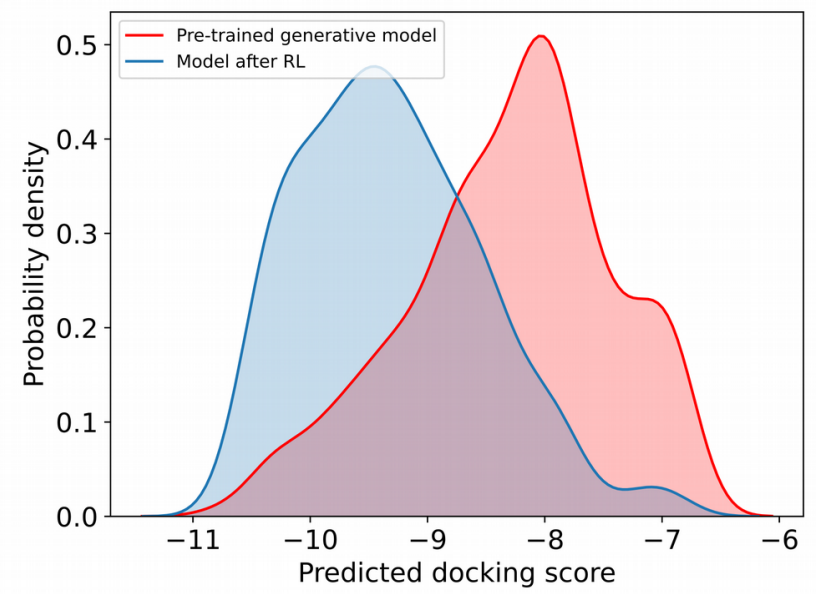

Figure S6: The maximum Tanimoto similarity distribution and the predicted docking score distribution for the generative model trained with only transfer learning (a and $\mathbf{b}$ ), with only reinforcement learning (c and $\mathbf{d}$ ). In comparison, when both transfer and reinforcement learning were used together, improvements in both the docking score and Tanimoto coefficient distributions could be observed (Fig. 2a and 2b of the manuscript). 


\section{References}

(1) ChEMBL Database, version 27, doi: 10.6019/CHEMBL.database.27 (accessed November 2019).

(2) Benhenda, M. ChemGAN Challenge for Drug Discovery: Can Al Reproduce Natural Chemical Diversity?, 2017; https://arxiv.org/abs/1708.08227.

(3) Brown, N.; Fiscato, M.; Segler, M. H. S.; Vaucher, A. C. GuacaMol: Benchmarking Models for De Novo Molecular Design. J. Chem. Inf. Model. 2019, 59, 1096-1108.

(4) Ertl, P.; Schuffenhauer, A. Estimation of Synthetic Accessibility Score of Drug-like Molecules based on Molecular Complexity and Fragment Contributions. $J$. Cheminform. 2009, 1, 8.

(5) Landrum, G. RDKit: Open-Source Cheminformatics Software. http://www.rdkit.org (accessed November 01, 2019).

(6) Ghoreschi, K.; Laurence, A.; O'Shea, J. J. Janus Kinases in Immune Cell Signaling. Immunol. Rev. 2009, 228, 273-287.

(7) Bousoik, E.; Aliabadi, H. M. "Do We Know Jack" About JAK? A Closer Look at JAK/STAT Signaling Pathway. Front. Oncol. 2018, 8, 287.

(8) Sievers, F.; Higgins, D. G. Clustal Omega, Accurate Alignment of Very Large Numbers of Sequences. Methods Mol. Biol. 2014, 1079, 105-16.

(9) UniProt Consortium. UniProt: A Worldwide Hub of Protein Knowledge. Nucleic Acids Res. 2019, 47, D506-D515.

(10) Velázquez, N. L. A.; Boggon, T. J. The Use of Structural Biology In Janus Kinase Targeted Drug Discovery. Curr. Drug Targets. 2011, 12, 546-555.

(11) Haan, C.; Behrmann, I.; Haan, S. Perspectives For the Use of Structural Information and Chemical Genetics to Develop Inhibitors of Janus Kinases. J. Cell Mol. Med. 2010, 14, 504-527.

(12) Goedken, E. R.; Argiriadi, M. A.; Banach, D. L.; Fiamengo, B. A.; Foley, S. E.; Frank, K. E.; George, J. S.; Harris, C. M.; Hobson, A. D.; Ihle, D. C.; Marcotte, D.; Merta, P. J.; Michalak, M. E.; Murdock, S. E.; Tomlinson, M. E.; Voss, J. W. Tricyclic Covalent Inhibitors Selectively Target Jak3 through an Active Site Thiol. J. Biol. Chem. 2015, 290, 4573-89.

(13) Telliez, J.; Dowty, M. E.; Wang, L.; Jussif, J.; Lin, T.; Li, L.; Moy, E.; Balbo, P.; Li, W.; Zhao, Y.; Crouse, K.; Dickinson, C.; Symanowicz, P.; Hegen, M.; Banker, M. E.; Vincent, F.; Unwalla, R.; Liang, S.; Gilbert, A. M.; Brown, M. F.; Hayward, M.; Montgomery, J.; Yang, X.; Bauman, J.; Trujillo, J. I.; Casimiro-Garcia, A.; Vajdos, F. F.; Leung, L.; Geoghegan, K. F.; Quazi, A.; Xuan, D.; Jones, L.; Hett, E.; Wright, K.; Clark, J. D.; Thorarensen, A. Discovery of a JAK3-selective inhibitor: Functional Differentiation of JAK3-Selective Inhibition Over Pan-JAK or JAK1-Selective Inhibition. ACS Chem. Biol. 2016, 11, 3442-3451.

(14) Pei, H.; He, L.; Shao, M.; Yang, Z.; Ran, Y.; Li, D.; Zhou, Y.; Tang, M.; Wang, T.; Gong, Y.; Chen, X.; Yang, S.; Xiang, M.; Chen, L. Discovery of a Highly Selective JAK3 Inhibitor for the Treatment of Rheumatoid Arthritis. Sci. Rep. 2018, 8, 5273.

(15) Dymock, B. W.; See, C. S. Inhibitors of JAK2 and JAK3: An Update on the Patent Literature 2010 - 2012. Expert Opin. Ther. Pat. 2013, 23, 449-501.

(16) Dymock, B. W.; Yang, E. G.; Chu-Farseeva, Y.; Yao, L. Selective JAK Inhibitors. Future Med. Chem. 2014, 6, 1439-1471.

(17) Douglas, J. J.; Cole, K. P.; Stephenson, C. R. J. Photoredox Catalysis in a Complex Pharmaceutical Setting: Toward the Preparation of JAK2 Inhibitor LY2784544. J. Org. Chem. 2014, 79, 11631-11643.

(18) Lin, T. E.; HuangFu, W.; Chao, M.; Sung, T.; Chang, C.; Chen, Y.; Hsieh, J.; Tu, H.; Huang, H.; Pan, S.; Hsu, K. A Novel Selective JAK2 Inhibitor Identified Using Pharmacological Interactions. Front. Pharmacol. 2018, 9, 1379. 
(19) Hanan, E. J.; van Abbema, A.; Barrett, K.; Blair, W. S.; Blaney, J.; Chang, C.; Eigenbrot, C.; Flynn, S.; Gibbons, P.; Hurley, C. A.; Kenny, J. R.; Kulagowski, J.; Lee, L.; Magnuson, S. R.; Morris, C.; Murray, J.; Pastor, R. M.; Rawson, T.; Siu, M.; Ultsch, M.; Zhou, A.; Sampath, D.; Lyssikatos, J. P. Discovery of Potent and Selective Pyrazolopyrimidine Janus Kinase 2 Inhibitors. J. Med. Chem. 2012, 55, 10090-10107.

(20) Su, Q.; Ioannidis, S.; Chuaqui, C.; Almeida, L.; Alimzhanov, M.; Bebernitz, G.; Bell, K.; Block, M.; Howard, T.; Huang, S.; Huszar, D.; Read, J. A.; Costa, C. R.; Shi, J.; Su, M.; Ye, M.; Zinda, M. Discovery of 1-methyl-1H-imidazole Derivatives as Potent Jak2 Inhibitors. J. Med. Chem. 2014, 57, 144-158.

(21) Ma, L.; Clayton, J. R.; Walgren, R. A.; Zhao, B.; Evans, R. J.; Smith, M. C.; HeinzTaheny, K. M.; Kreklau, E. L.; Bloem, L.; Pitou, C.; Shen, W.; Strelow, J. M.; Halstead, C.; Rempala, M. E.; Parthasarathy, S.; Gillig, J. R.; Heinz, L. J.; Pei, H.; Wang, Y.; Stancato, L. F.; Dowless, M. S.; Iversen, P. W.; Burkholder, T. P. Discovery and Characterization of LY2784544, a Small-Molecule Tyrosine Kinase Inhibitor of JAK2V617F. Blood Cancer Journal. 2013, 3, e109.

(22) Ambit Biosciences Corp. 2-cycloquinazoline Derivatives and Methods of Use thereof. WO2012030910; 2012.

(23) Ambit Biosciences Corp. Quinoline and Isoquinoline Compounds and Methods of Use thereof. WO2012030944; 2012.

(24) Cephalon, Inc. Preparation and Uses of 1,2,4-triazolo[1,5-a]pyridine Derivatives. WO2010141796; 2010.

(25) AstraZeneca AB. Heterocyclic JAK Kinase Inhibitors. WO2010038060; 2010.

(26) Genentech, Inc. Pyrazolopyrimidine JAK Inhibitor Compounds and Methods. WO2010051549; 2010.

(27) AstraZeneca AB. 2-(imidazolylamino)- pyridine Derivatives and their Use as JAK Kinase Inhibitors. WO2010020810; 2010.

(28) Ambit Biosciences Corp. JAK Kinase Modulating Quinazoline Derivatives and Methods of Use thereof. WO2010099379; 2010.

(29) Purandare, A. V.; McDevitt, T. M.; Wan, H.; You, D.; Penhallow, B.; Han, X.; Vuppugalla, R.; Zhang, Y.; Ruepp, S. U.; Trainor, G. L.; Lombardo, L.; Pedicord, D.; Gottardis, M. M.; Ross-Macdonald, P.; de Silva, H.; Hosbach, J.; Emanuel, S. L.; Blat, Y.; Fitzpatrick, E.; Taylor, T. L.; McIntyre, K. W.; Michaud, E.; Mulligan, C.; Lee, F. Y.; Woolfson, A.; Lasho, T. L.; Pardanani, A.; Tefferi, A.; Lorenzi, M. V. Characterization of BMS-911543, a Functionally Selective Small-Molecule Inhibitor of JAK2. Leukemia. 2012, 26, 280-8.

(30) Lim, J.; Taoka, B.; Otte, R. D.; Spencer, K.; Dinsmore, C. J.; Altman, M. D.; Chan, G.; Rosenstein, C.; Sharma, S.; Su, H.; Szewczak, A. A.; Xu, L.; Yin, H.; ZugayMurphy, J.; Marshall, C. G.; Young, J. R. Discovery of 1-amino-5H-pyrido[4,3b]indol-4-carboxamide inhibitors of Janus kinase 2 (JAK2) for the treatment of myeloproliferative disorders. J. Med. Chem. 2011, 54, 7334-7349.

(31) Bemis, G. W.; Murcko, M. A. The Properties of Known Drugs. 1. Molecular Frameworks. J. Med. Chem. 1996, 39, 2887-93.

(32) Shang, J.; Sun, H.; Liu, H.; Chen, F.; Tian, S.; Pan, P.; Li, D.; Kong, D.; Hou, T. Comparative Analyses of Structural Features and Scaffold Diversity for Purchasable Compound Libraries. J. Cheminform. 2017, 9, 25.

(33) Butina, D. Unsupervised Data Base Clustering Based on Daylight's Fingerprint and Tanimoto Similarity: A Fast and Automated Way To Cluster Small and Large Data Sets. J. Chem. Inf. Comput. Sci. 1999, 39, 747-750.

(34) O'Hagan, S.; Kell, D. B. MetMaxStruct: A Tversky-Similarity-Based Strategy for Analysing the (Sub)Structural Similarities of Drugs and Endogenous Metabolites. Front. Pharmacol. 2016, 7, 266. 
(35) Bickerton, G. R.; Paolini, G. V.; Besnard, J.; Muresan, S.; Hopkins, A. L. Quantifying the Chemical Beauty of Drugs. Nat. Chem. 2012, 4, 90-98. 